\title{
MAXIMAL OPERATORS OF SCHRÖDINGER TYPE WITH A COMPLEX PARAMETER
}

\author{
PER SJÖLIN
}

\begin{abstract}
Maximal operators of Schrödinger type but with a complex parameter are considered. For these operators we obtain results which in a certain sense lie between the results for the corresponding maximal operators for solutions to the Schrödinger equation and for solutions to the heat equation.
\end{abstract}

\section{Introduction}

Letting $f$ belong to the Schwartz space $\mathscr{S}(\mathrm{R})$ we set

$$
S_{t} f(x)=\int_{\mathrm{R}} e^{i x \xi} e^{i t \xi^{2}} \widehat{f}(\xi) d \xi, \quad x \in \mathrm{R} .
$$

Here $t$ is a complex number with $\operatorname{Im} t \geq 0$ and $\widehat{f}$ denotes the Fourier transform of $f$, defined by

$$
\widehat{f}(\xi)=\int_{\mathrm{R}} e^{-i \xi x} f(x) d x .
$$

We also set $U(x, t)=(2 \pi)^{-1} S_{t} f(x)$ for $x \in \mathrm{R}$ and $t \in \mathrm{R}$. It then follows that $U(x, 0)=f(x)$ and $U$ satisfies the Schrödinger equation $i \partial U / \partial t=$ $\partial^{2} U / \partial x^{2}$.

We also introduce the maximal function $S^{*} f$ defined by

$$
S^{*} f(x)=\sup _{0<t<1}\left|S_{t} f(x)\right|, \quad x \in \mathrm{R},
$$

and define Sobolev spaces $H_{s}$ by setting

$$
H_{s}=\left\{f \in \mathscr{S}^{\prime} ;\|f\|_{H_{s}}<\infty\right\}, \quad s \in \mathrm{R},
$$

where

$$
\|f\|_{H_{s}}=\left(\int_{\mathrm{R}}\left(1+\xi^{2}\right)^{s}|\widehat{f}(\xi)|^{2} d \xi\right)^{1 / 2} .
$$

Received January 21, 2008. 
It is well-known that the estimate

$$
\left\|S^{*} f\right\|_{2} \leq C\|f\|_{H_{s}}
$$

holds for $s>1 / 2$ and does not hold for $s<1 / 2$ (see [2]). Here $\left\|S^{*} f\right\|_{2}$ denotes the norm of $S^{*} f$ in the space $L^{2}(\mathrm{R})$. We then set

$$
H_{u} f(x)=S_{i u} f(x)=\int_{\mathrm{R}} e^{i x \xi} e^{-u \xi^{2}} \widehat{f}(\xi) d \xi, \quad x \in \mathrm{R},
$$

for $u \geq 0$. If we set $V(x, u)=(2 \pi)^{-1} H_{u} f(x)$ for $x \in \mathrm{R}$ and $u \geq 0$, then $V(x, 0)=f(x)$ and $V$ satisfies the heat equation $\partial V / \partial u=\partial^{2} V / \partial x^{2}$. We also introduce the maximal function $H^{*} f$ defined by

$$
H^{*} f(x)=\sup _{0<u<1}\left|H_{u} f(x)\right|, \quad x \in \mathrm{R} .
$$

It is then well-known that the estimate $\left\|H^{*} f\right\|_{2} \leq C\|f\|_{H_{s}}$ holds if and only if $s \geq 0$.

We shall then introduce a class of maximal operators for which one has results lying between the above results for $S^{*}$ and $H^{*}$. For $0<\gamma<\infty$ we set

$$
P_{u} f(x)=S_{u+i u^{\gamma}} f(x)=\int_{\mathrm{R}} e^{i x \xi} e^{i u \xi^{2}} e^{-u^{\gamma} \xi^{2}} \widehat{f}(\xi) d \xi, \quad x \in \mathrm{R}, \quad 0<u<1,
$$

and

$$
P^{*} f(x)=\sup _{0<u<1}\left|P_{u} f(x)\right|, \quad x \in \mathbf{R} .
$$

We shall here study the inequality

$$
\left\|P^{*} f\right\|_{2} \leq C\|f\|_{H_{s}}
$$

for various values of $\gamma$. We have the following results.

THEOREM 1.

(i) For $0<\gamma \leq 1$ (1) holds if and only if $s \geq 0$.

(ii) For $\gamma=2$ (1) holds if and only if $s \geq 1 / 4$.

(iii) If $\gamma \geq 4$ and (1) holds then $s \geq 1 / 2-1 / \gamma$.

For $\gamma>0$ we let $E_{\gamma}$ denote the set of all $s$ with the property that (1) holds. Also set $s(\gamma)=\inf E_{\gamma}$ for $\gamma>0$. Using the fact that $\lim _{u \rightarrow 0} P_{u} f(x)=$ $2 \pi f(x)$ it is easy to see that $s(\gamma) \geq 0$. We shall use the following lemma.

Lemma 1. Assume that $g$ and $h$ are continuous functions on the interval $(0,1)$ and that $0 \leq g(u) \leq h(u)$ for $0<u<1$. Set

$$
P_{g}^{*} f(x)=\sup _{0<u<1}\left|S_{u+i g(u)} f(x)\right|
$$


and

$$
P_{h}^{*} f(x)=\sup _{0<u<1}\left|S_{u+i h(u)} f(x)\right| .
$$

Then

$$
\left\|P_{h}^{*} f\right\|_{2} \leq C\left\|P_{g}^{*} f\right\|_{2} .
$$

It follows from the lemma that $s(\gamma)$ is an increasing function of $\gamma$ on the interval $(0, \infty)$. Also the result mentioned above for the operator $S^{*}$ implies that $s(\gamma) \leq 1 / 2$ (take $g(u) \equiv 0$ and $h(u)=u^{\gamma}$ in the lemma). The results in Theorem 1 can then be stated in the following way.

THEOREM 2.

(i) For $0<\gamma \leq 1$ one has $s(\gamma)=0$.

(ii) $s(2)=1 / 4$.

(iii) For $\gamma>4$ one has $1 / 2-1 / \gamma \leq s(\gamma) \leq 1 / 2$ and hence $\lim _{\gamma \rightarrow \infty} s(\gamma)=$ $1 / 2$.

In Section 2 we shall prove Lemma 1 and state and prove a second lemma. In Section 3 we shall prove Theorem 1.

AcKnowledgement. A question by Håkan Hedenmalm drew my attention to the problem studied in this paper.

\section{Lemmas}

Proof of Lemma 1. We have

$$
\begin{aligned}
S_{u+i h(u)} f(x) & =\int e^{i x \xi} e^{i u \xi^{2}} e^{-h(u) \xi^{2}} \widehat{f}(\xi) d \xi \\
& =\int e^{i x \xi} e^{i u \xi^{2}} e^{-g(u) \xi^{2}} e^{-(h(u)-g(u)) \xi^{2}} \widehat{f}(\xi) d \xi \\
& =\int e^{i x \xi} e^{i u \xi^{2}} e^{-g(u) \xi^{2}} e^{-v \xi^{2}} \widehat{f}(\xi) d \xi
\end{aligned}
$$

where $v=h(u)-g(u) \geq 0$.

For $v=0$ one has

$$
S_{u+i h(u)} f(x)=S_{u+i g(u)} f(x) .
$$

We then assume $v>0$. It is well-known that

$$
e^{-v \xi^{2}}=\widehat{K}_{v}(\xi)=\int e^{-i \xi y} K_{v}(y) d y,
$$


where

$$
K_{v}(y)=\frac{1}{v^{1 / 2}} \frac{1}{2 \sqrt{\pi}} e^{-y^{2} /(4 v)}=\frac{1}{v^{1 / 2}} K\left(y / v^{1 / 2}\right)
$$

with $K(y)=e^{-y^{2} / 4} /(2 \sqrt{\pi})$. It follows that

$$
\begin{aligned}
S_{u+i h(u)} f(x) & =\int e^{i x \xi} e^{i u \xi^{2}} e^{-g(u) \xi^{2}}\left(\int e^{-i \xi y} K_{v}(y) d y\right) \widehat{f}(\xi) d \xi \\
& =\int\left(\int e^{i(x-y) \xi} e^{i u \xi^{2}} e^{-g(u) \xi^{2}} \widehat{f}(\xi) d \xi\right) K_{v}(y) d y \\
& =\int S_{u+i g(u)} f(x-y) K_{v}(y) d y=K_{v} * S_{u+i g(u)} f(x)
\end{aligned}
$$

and hence

$$
\left|S_{u+i h(u)} f(x)\right| \leq K_{v} * P_{g}^{*} f(x) \leq C M P_{g}^{*} f(x)
$$

where $M$ denotes the Hardy-Littlewood maximal operator.

We conclude that

$$
P_{h}^{*} f(x) \leq P_{g}^{*} f(x)+C M P_{g}^{*} f(x)
$$

and since $M$ is a bounded operator on $L^{2}(\mathrm{R})$ we obtain $\left\|P_{h}^{*} f\right\|_{2} \leq C\left\|P_{g}^{*} f\right\|_{2}$.

The following lemma was proved in [3].

Lemma 2. Assume that $a>1,1 / 2 \leq s<1$ and $\mu \in C_{0}^{\infty}(\mathrm{R})$. Then

$$
\left.\left|\int_{\mathrm{R}} e^{i x \xi+i t|\xi|^{a}}\right| \xi\right|^{-s} \mu(\xi / N) d \xi \mid \leq C \frac{1}{|x|^{1-s}}
$$

for $x \in \mathrm{R}, t \in \mathrm{R}$ and $N=1,2,3, \ldots$. Here the constant $C$ may depend on $s$ and a but not on $x, t$ or $N$.

The next lemma will be used to prove that $s(2) \leq 1 / 4$.

Lemma 3. Assume that $1 / 2 \leq \alpha<1,0<d_{1}<1,0<d_{2}<1$, and $\mu \in C_{0}^{\infty}(\mathrm{R})$ and $\mu$ even and real-valued. Then

$$
\left|\int_{\mathrm{R}} \frac{e^{i\left(\left(d_{1}-d_{2}\right) \xi^{2}-x \xi\right)}}{\left(1+\xi^{2}\right)^{\alpha / 2}} e^{-\left(d_{1}^{2}+d_{2}^{2}\right) \xi^{2}} \mu(\xi / N) d \xi\right| \leq K(x)
$$

for $x \in \mathrm{R}$ and $N=1,2,3, \ldots$, where $K \in L^{1}(\mathrm{R})$. Here $K$ is independent of $d_{1}$, $d_{2}$ and $N$, and one may take $K(x)=C x^{-2}$ for $|x| \geq C_{0}$ and $K(x)=C|x|^{\alpha-1}$ for $|x|<C_{0}$. Here $C_{0}$ denotes a large constant. 
Proof of Lemma 3. The structure in the proof of the lemma will be the same as in the proof of Lemma 3 in [4]. Without loss of generality we may assume $d_{2}<d_{1}$. We set $d=d_{1}-d_{2}$ and $\varepsilon=d_{1}^{2}+d_{2}^{2}$ so that $0<d<1$ and $0<\varepsilon<2$. First assume $|x| \geq C_{0}$ where $C_{0}$ denotes a large constant.

We choose an even function $\varphi_{0} \in C_{0}^{\infty}(\mathrm{R})$ such that $\varphi_{0}(\xi)=1$ for $|\xi| \leq 1 / 2$ and $\varphi_{0}(\xi)=0$ for $|\xi| \geq 1$.

Set

$$
\psi(\xi)=\left(1+\xi^{2}\right)^{-\alpha / 2} e^{-\varepsilon \xi^{2}} \mu(\xi / N)
$$

and $\psi_{0}=\psi \varphi_{0}$ so that supp $\psi_{0} \subset[-1,1]$.

We also set $\rho=|x| /(2 d)$ and let $K$ denote a large constant. Then choose $\varphi_{2} \in C_{0}^{\infty}(\mathrm{R})$ so that $\operatorname{supp} \varphi_{2} \subset[\rho / 4,2 K \rho]$ and $\varphi_{2}(\xi)=1$ for $\rho / 2 \leq \xi \leq K \rho$. We may also assume that $\left|\varphi_{2}^{\prime}(\xi)\right| \leq C \xi^{-1}$ and $\left|\varphi_{2}^{\prime \prime}(\xi)\right| \leq C \xi^{-2}$ for $\xi>0$. We then set $\varphi_{3}=\left(1-\varphi_{2}\right) \chi_{[K \rho, \infty)}$ and $\varphi_{1}=\left(1-\varphi_{2}-\varphi_{0}\right) \chi_{[0, \rho / 2]}$.

Let $\check{\varphi}$ be defined by $\check{\varphi}(\xi)=\varphi(-\xi)$ and set $\varphi_{-1}=\check{\varphi}_{1}, \varphi_{-2}=\check{\varphi}_{2}$ and $\varphi_{-3}=\check{\varphi}_{3}$. Setting $F(\xi)=d \xi^{2}-x \xi$ we then have

$$
\int_{-\infty}^{\infty} e^{i F} \psi d \xi=\sum_{j=0}^{3} \int e^{i F} \psi \varphi_{j} d \xi+\sum_{j=1}^{3} \int e^{i F} \psi \varphi_{-j} d \xi .
$$

The integrals $\int e^{i F} \psi \varphi_{-j}$ can be reduced to $\int e^{i F} \psi \varphi_{j}$ for $j=1,2$, 3. Setting $\psi_{j}=\psi \varphi_{j}, j=0,1,2,3$, it is therefore sufficient to estimate the integrals

$$
J_{j}=\int e^{i F} \psi_{j} d \xi, \quad j=0,1,2,3 .
$$

We claim that one has the following estimates for $j=1,2,3$ and $\xi \geq 1 / 2$ :

$$
\left|\psi_{j}(\xi)\right| \leq C \frac{1}{\left(1+\xi^{2}\right)^{\alpha / 2}},
$$

$$
\left|\psi_{j}^{\prime}(\xi)\right| \leq C \frac{1}{\left(1+\xi^{2}\right)^{\alpha / 2} \xi},
$$

and

$$
\left|\psi_{j}^{\prime \prime}(\xi)\right| \leq C \frac{1}{\left(1+\xi^{2}\right)^{\alpha / 2} \xi^{2}} .
$$

We $\operatorname{set} h(\xi)=h_{\varepsilon}(\xi)=e^{-\varepsilon \xi^{2}}$ for $\xi \geq 1 / 2$ and $0<\varepsilon<2$. The above estimates for $\psi_{j}, \psi_{j}^{\prime}$ and $\psi_{j}^{\prime \prime}$ will follow if we can prove that

$$
\left|h^{\prime}(\xi)\right| \leq C \frac{1}{\xi}, \quad \xi \geq 1 / 2,
$$


and

$$
\left|h^{\prime \prime}(\xi)\right| \leq C \frac{1}{\xi^{2}}, \quad \xi \geq 1 / 2,
$$

with $C$ independent of $\varepsilon$.

We have

$$
h^{\prime}(\xi)=-e^{-\varepsilon \xi^{2}} 2 \varepsilon \xi
$$

and

$$
h^{\prime \prime}(\xi)=-e^{-\varepsilon \xi^{2}} 2 \varepsilon+e^{-\varepsilon \xi^{2}} 4 \varepsilon^{2} \xi^{2} .
$$

It follows that for $\xi \geq 1 / 2$ one has

$$
\left|h^{\prime}(\xi)\right| \leq e^{-\varepsilon \xi^{2}} 2 \varepsilon \xi=e^{-\varepsilon \xi^{2}} 2 \varepsilon \xi^{2} \frac{1}{\xi} \leq 2\left(\max _{t>0} t e^{-t}\right) \frac{1}{\xi}=2 A \frac{1}{\xi},
$$

where $A=\max _{t \geq 0} t e^{-t}$. Also

$$
\begin{aligned}
\left|h^{\prime \prime}(\xi)\right| \leq e^{-\varepsilon \xi^{2}} 2 \varepsilon+4 e^{-\varepsilon \xi^{2}} \varepsilon^{2} \xi^{2} & \\
& =e^{-\varepsilon \xi^{2}} 2 \varepsilon \xi^{2} \frac{1}{\xi^{2}}+4 e^{-\varepsilon \xi^{2}} \varepsilon^{2} \xi^{4} \frac{1}{\xi^{2}} \leq 2 A \frac{1}{\xi^{2}}+4 B \frac{1}{\xi^{2}},
\end{aligned}
$$

where $B=\max _{t \geq 0} t^{2} e^{-t}$. Hence (5) and (6) are proved and (2), (3) and (4) follow.

We shall first estimate $J_{0}$. We have

$$
J_{0}=\int e^{-i x \xi} e^{i d \xi^{2}} \psi_{0}(\xi) d \xi
$$

where supp $\psi_{0} \subset[-1,1]$ and two integrations by parts give the estimate $\left|J_{0}\right| \leq C x^{-2}$.

We shall then estimate $J_{2}$. One has

$$
J_{2}=\int e^{i F} \psi_{2} d \xi
$$

where

$$
\psi_{2}(\xi)=\left(1+\xi^{2}\right)^{-\alpha / 2} e^{-\varepsilon \xi^{2}} \mu(\xi / N) \varphi_{2}(\xi)
$$

and supp $\psi_{2} \subset[\rho / 4,2 K \rho]$.

We have $F^{\prime \prime}(\xi)=2 d$ and van der Corput's Lemma with the second derivative (see Stein [5], p. 334) gives

$$
\left[J_{2} \mid \leq C d^{-1 / 2}\left(\max \left|\psi_{2}\right|+\int\left|\psi_{2}^{\prime}\right| d \xi\right) .\right.
$$


It is clear that

$$
\max \left|\psi_{2}\right| \leq C \rho^{-\alpha} e^{-c \varepsilon \rho^{2}}
$$

where $c$ denotes a positive constant.

We also set

$$
v(\xi)=\left(1+\xi^{2}\right)^{-\alpha / 2} \mu(\xi / N) \varphi_{2}(\xi)
$$

so that

$$
\psi_{2}(\xi)=v(\xi) e^{-\varepsilon \xi^{2}}
$$

and

$$
\psi_{2}^{\prime}(\xi)=v(\xi)\left(\frac{d}{d \xi} e^{-\varepsilon \xi^{2}}\right)+v^{\prime}(\xi) e^{-\varepsilon \xi^{2}} .
$$

It follows that

$$
\begin{aligned}
\int\left|\psi_{2}^{\prime}(\xi)\right| d \xi & \leq \int\left|v(\xi)\left(\frac{d}{d \xi} e^{-\varepsilon \xi^{2}}\right)\right| d \xi+\int\left|v^{\prime}(\xi) e^{-\varepsilon \xi^{2}}\right| d \xi \\
& \leq C \rho^{-\alpha} \int_{\rho / 4}^{2 K \rho}\left|\frac{d}{d \xi} e^{-\varepsilon \xi^{2}}\right| d \xi+\int_{\rho / 4}^{2 K \rho} \rho^{-\alpha-1} d \xi e^{-c \varepsilon \rho^{2}} \\
& \leq C \rho^{-\alpha} \int_{\rho / 4}^{2 K \rho}-\left(\frac{d}{d \xi} e^{-\varepsilon \xi^{2}}\right) d \xi+C \rho^{-\alpha} e^{-c \varepsilon \xi^{2}} \\
& =-C \rho^{-\alpha}\left[e^{-\varepsilon \xi^{2}}\right]_{\rho / 4}^{2 K \rho}+C \rho^{-\alpha} e^{-c \varepsilon \rho^{2}} \leq C \rho^{-\alpha} e^{-c \varepsilon \rho^{2}}
\end{aligned}
$$

Combining this estimate with (7) and (8) we obtain

$$
\begin{aligned}
\left|J_{2}\right| \leq C d^{-1 / 2} \rho^{-\alpha} e^{-c \varepsilon \rho^{2}}=C d^{-1 / 2}\left(\frac{|x|}{d}\right)^{-\alpha} & e^{-c \varepsilon x^{2} / d^{2}} \\
& \leq C d^{\alpha-1 / 2}|x|^{-\alpha} e^{-c x^{2}} \leq C e^{-c x^{2}},
\end{aligned}
$$

since $\alpha \geq 1 / 2$ and $d^{2} \leq \varepsilon$. In fact

$$
d^{2}=\left(d_{1}-d_{2}\right)^{2}=d_{1}^{2}+d_{2}^{2}-2 d_{1} d_{2} \leq d_{1}^{2}+d_{2}^{2}=\varepsilon .
$$

This concludes the estimate of $J_{2}$ and we shall then estimate $J_{1}$. One has

$$
J_{1}=\int e^{i F} \psi_{1} d \xi
$$

where supp $\psi_{1} \subset[1 / 2, \rho / 2]$ and $F^{\prime}=2 d \xi-x$.

On the interval $[1 / 2, \rho / 2]$ one has

$$
2 d \xi \leq 2 d \rho / 2=d \rho=d \frac{|x|}{2 d}=\frac{|x|}{2} \quad \text { and } \quad\left|F^{\prime}\right| \geq|x| / 2 \geq 2 d \xi
$$


Also $F^{\prime \prime}=2 d$ and $F^{(3)}=0$. It follows that $\left|F^{\prime \prime}\right| /\left|F^{\prime}\right| \leq C \xi^{-1}$ for $1 / 2 \leq \xi \leq$ $\rho / 2$. Integrating by parts twice we obtain

$$
\begin{aligned}
J_{1}=\int e^{i F} \psi_{1} d \xi= & \int i F^{\prime} e^{i F} \frac{\psi_{1}}{i F^{\prime}} d \xi \\
= & -\int e^{i F}\left(\frac{1}{i F^{\prime}} \psi_{1}^{\prime}-\frac{1}{i} \frac{F^{\prime \prime}}{\left(F^{\prime}\right)^{2}} \psi_{1}\right) d \xi \\
= & -\int i F^{\prime} e^{i F}\left(\frac{1}{\left(i F^{\prime}\right)^{2}} \psi_{1}^{\prime}+\frac{F^{\prime \prime}}{\left(F^{\prime}\right)^{3}} \psi_{1}\right) d \xi \\
= & \int e^{i F}\left(\frac{1}{i^{2}} \frac{1}{\left(F^{\prime}\right)^{2}} \psi_{1}^{\prime \prime}-\frac{1}{i^{2}} \frac{2 F^{\prime \prime}}{\left(F^{\prime}\right)^{3}} \psi_{1}^{\prime}\right. \\
& \left.\quad+\frac{F^{\prime \prime}}{\left(F^{\prime}\right)^{3}} \psi_{1}^{\prime}+\frac{F^{(3)}}{\left(F^{\prime}\right)^{3}} \psi_{1}-\frac{3\left(F^{\prime \prime}\right)^{2}}{\left(F^{\prime}\right)^{4}} \psi_{1}\right) d \xi
\end{aligned}
$$

and hence

$$
\begin{aligned}
\left|J_{1}\right| & \leq C \int \frac{1}{\left|F^{\prime}\right|^{2}}\left(\left|\psi_{1}^{\prime \prime}\right|+\frac{\left|F^{\prime \prime}\right|}{\left|F^{\prime}\right|}\left|\psi_{1}^{\prime}\right|+\frac{\left|F^{\prime \prime}\right|^{2}}{\left|F^{\prime}\right|^{2}}\left|\psi_{1}\right|\right) d \xi \\
& \leq C \frac{1}{x^{2}} \int_{1 / 2}^{\infty} \xi^{-\alpha-2} d \xi=C \frac{1}{x^{2}} .
\end{aligned}
$$

It remains to estimate $J_{3}=\int e^{i F} \psi_{3} d \xi$. Here supp $\psi_{3} \subset[K \rho, \infty)$. For

$$
\xi \geq K \rho=K \frac{|x|}{2 d}
$$

we have

$$
2 d \xi \geq 2 d K \frac{|x|}{2 d}=K|x|
$$

and hence $\left|F^{\prime}\right| \geq c|x|$ and $\left|F^{\prime}\right| \geq c d \xi$.

We can estimate $J_{3}$ in the same way as we estimated $J_{1}$. We can use the inequality (9) with $\psi_{1}$ replaces by $\psi_{3}$ and $J_{1}$ replaced by $J_{3}$. One obtains $\left|J_{3}\right| \leq C|x|^{-2}$.

We have proved Lemma 3 in the case $|x| \geq C_{0}$. It remains to study the case $|x|<C_{0}$. The estimate in this case follows from the proof in [3] of our Lemma 2. The proof of Lemma 3 is complete. 


\section{Proof of Theorem 1}

Proof of Theorem 1. We shall first study the case $0<\gamma \leq 1$. It is wellknown that $e^{-a x^{2}}$ has Fourier transform $\sqrt{\pi} a^{-1 / 2} e^{-\xi^{2} / 4 a}$ for $a>0$. For Re $z>$ 0 we then set $z^{1 / 2}=|z|^{1 / 2} e^{i(\arg z) / 2}$ where $-\pi / 2<\arg z<\pi / 2$. By use of elementary properties of analytic functions it is then easy to prove that $e^{-z x^{2}}$ has Fourier transform

$$
\frac{\sqrt{\pi}}{z^{1 / 2}} e^{-\xi^{2} / 4 z}
$$

for $\operatorname{Re} z>0$. It also follows that $e^{-z \xi^{2}}$ is the Fourier transform of

$$
\frac{1}{2 \sqrt{\pi} z^{1 / 2}} e^{-x^{2} / 4 z}
$$

for $\operatorname{Re} z>0$.

Setting $t=u+i v$ with $u>0, v>0$ we then have $e^{i t \xi^{2}}=e^{i(u+i v) \xi^{2}}=$ $e^{-(v-i u) \xi^{2}}$. Taking $z=v-i u$ in (10) it then follows that $e^{-(v-i u) \xi^{2}}$ is the Fourier transform of

$$
K(x)=\frac{1}{2 \sqrt{\pi}(v-i u)^{1 / 2}} e^{-x^{2} /(4(v-i u))} .
$$

It is clear that

$$
|K(x)| \leq \frac{1}{\left(v^{2}+u^{2}\right)^{1 / 4}}\left|e^{-x^{2} /(4(v-i u))}\right| .
$$

Since

$$
\frac{1}{v-i u}=\frac{v}{v^{2}+u^{2}}+i \frac{u}{v^{2}+u^{2}}
$$

we conclude that

$$
|K(x)| \leq \frac{1}{\left(v^{2}+u^{2}\right)^{1 / 4}} e^{-x^{2} v /\left(4\left(v^{2}+u^{2}\right)\right)} .
$$

Letting $0<u<1$ and $v=u^{\gamma}$ with $0<\gamma \leq 1$ we then obtain

$$
\begin{aligned}
|K(x)| & \leq \frac{1}{\left(u^{2 \gamma}+u^{2}\right)^{1 / 4}} \exp \left(-\frac{x^{2}}{4} \frac{u^{\gamma}}{u^{2 \gamma}+u^{2}}\right) \\
& \leq \frac{1}{u^{\gamma / 2}} e^{-c x^{2} / u^{\gamma}}=\frac{1}{u^{\gamma / 2}} L\left(x / u^{\gamma / 2}\right),
\end{aligned}
$$

where $c>0$ and $L(x)=e^{-c x^{2}}$. It follows that $P^{*} f(x) \leq C M f(x)$ and hence the first part of Theorem 1 follows. 
We shall then study the case $\gamma=2$. We let $u(x)$ denote a measurable function on $\mathrm{R}$ with $0<u(x)<1$ and set

$$
P f(x)=\int_{\mathrm{R}} e^{i x \xi} e^{i u(x) \xi^{2}} e^{-u(x)^{2} \xi^{2}} \widehat{f}(\xi) d \xi, \quad x \in \mathrm{R} .
$$

We have to prove that

$$
\|P f\|_{2} \leq C\|f\|_{H_{s}}=C\left(\int_{\mathrm{R}}|\widehat{f}(\xi)|^{2}\left(1+\xi^{2}\right)^{s} d \xi\right)^{1 / 2}
$$

for $s \geq 1 / 4$. We may also assume $s<1 / 2$.

Setting $g(\xi)=\widehat{f}(\xi)\left(1+\xi^{2}\right)^{s / 2}$ and defining $T$ by

$$
T g(x)=\int_{\mathrm{R}} e^{i x \xi} e^{i u(x) \xi^{2}} e^{-u(x)^{2} \xi^{2}}\left(1+\xi^{2}\right)^{-s / 2} g(\xi) d \xi
$$

we have $P f(x)=T g(x)$. It is therefore sufficient to prove that $\|T g\|_{2} \leq$ $C\|g\|_{2}$. For $N=1,2,3, \ldots$ set

$$
T_{N} g(x)=\chi_{N}(x) \int_{\mathrm{R}} e^{i x \xi} e^{i u(x) \xi^{2}} e^{-u(x)^{2} \xi^{2}}\left(1+\xi^{2}\right)^{-s / 2} \rho_{N}(\xi) g(\xi) d \xi
$$

Here $\chi_{N}(x)=\chi(x / N)$ and $\rho_{N}(\xi)=\rho(\xi / N)$ and $\chi$ and $\rho \in C_{0}^{\infty}(\mathrm{R})$ and have the property that $\chi(x)=\rho(x)=1$ for $|x| \leq 1$ and $\chi(x)=\rho(x)=0$ for $|x| \geq 2$. We also assume that $\chi$ and $\rho$ are even and real-valued. It is sufficient to prove that

$$
\left\|T_{N} g\right\|_{2} \leq C\|g\|_{2}, \quad N=1,2,3, \ldots
$$

It is clear that

$$
T_{N}^{*} h(\xi)=\rho_{N}(\xi)\left(1+\xi^{2}\right)^{-s / 2} \int_{\mathrm{R}} e^{-i x \xi} e^{-i u(x) \xi^{2}} e^{-u(x)^{2} \xi^{2}} \chi_{N}(x) h(x) d x
$$

and it is sufficient to prove that

$$
\left\|T_{N}^{*} h\right\|_{2} \leq C\|h\|_{2}, \quad N=1,2,3, \ldots
$$


Invoking Lemma 3 we now have

$$
\begin{aligned}
\left\|T_{N}^{*} h\right\|_{2}^{2} & \\
= & \int T_{N}^{*} h(\xi) \overline{T_{N}^{*} h(\xi)} d \xi \\
= & \int \rho_{N}(\xi)^{2}\left(1+\xi^{2}\right)^{-s}\left(\int e^{-i x \xi} e^{-i u(x) \xi^{2}} e^{-u(x)^{2} \xi^{2}} \chi_{N}(x) h(x) d x\right) \\
& \times\left(\int e^{i y \xi} e^{i u(y) \xi^{2}} e^{-u(y)^{2} \xi^{2}} \chi_{N}(y) \overline{h(y)} d y\right) \\
= & \int\left(\int\left(1+\xi^{2}\right)^{-s} e^{i(y-x) \xi} e^{i(u(y)-u(x)) \xi^{2}} e^{-\left(u(x)^{2}+u(y)^{2}\right) \xi^{2}} \mu(\xi / N) d \xi\right) \\
& \times \chi_{N}(x) \chi_{N}(y) h(x) \overline{h(y)} d x d y \\
\leq & C \iint K(x-y)|h(x)||h(y)| d x d y \leq C\|h\|_{2}^{2} .
\end{aligned}
$$

Here we have set $\mu=\rho^{2}$ and according to Lemma 3 we have $K \in L^{1}(\mathrm{R})$ since $1 / 4 \leq s<1 / 2$. Hence (12) and (11) are proved.

We shall then prove that if $\gamma=2$ and $s<1 / 4$ then (1) does not hold. First choose $g \in C_{0}^{\infty}(\mathrm{R})$ such that supp $g \subset(-1,1), g(\xi) \geq 0$ and $g(\xi)=1$ for $|\xi| \leq 1 / 2$. Then let $v>0$ denote a small number and define a function $f_{v}$ by setting

$$
\widehat{f_{v}}(\xi)=v g(v \xi+1 / v) .
$$

It is well-known and easy to prove that $\left\|f_{v}\right\|_{H_{s}} \rightarrow 0$ as $v \rightarrow 0$ if $s<1 / 4$ (see [1]). Setting $u=u(x)=x v^{2} / 2$ and assuming $0<x<1 / 100$ we have

$$
P_{u} f_{v}(x)=\int v g(v \xi+1 / v) e^{i x \xi} e^{i x v^{2} \xi^{2} / 2} e^{-x^{2} v^{4} \xi^{2} / 4} d \xi .
$$

In this integral we make a change of variable $\eta=v \xi+1 / v$, so that $d \eta=v d \xi$ and $\xi=\eta / v-1 / v^{2}$. One obtains

$$
P_{u} f_{v}(x)=\int g(\eta) e^{i F(\eta)} e^{G(\eta)} d \eta
$$

where

$$
F(\eta)=x\left(\frac{\eta}{v}-\frac{1}{v^{2}}\right)+\frac{x v^{2}}{2}\left(\frac{\eta}{v}-\frac{1}{v^{2}}\right)^{2}
$$

and

$$
G(\eta)=-\frac{x^{2} v^{4}}{4}\left(\frac{\eta}{v}-\frac{1}{v^{2}}\right)^{2}
$$


Hence

$$
\begin{aligned}
F(\eta) & =\frac{x \eta}{v}-\frac{x}{v^{2}}+\frac{x v^{2}}{2}\left(\frac{\eta^{2}}{v^{2}}+\frac{1}{v^{4}}-\frac{2 \eta}{v^{3}}\right) \\
& =\frac{x \eta}{v}-\frac{x}{v^{2}}+\frac{x \eta^{2}}{2}+\frac{x}{2 v^{2}}-\frac{x \eta}{v}=\frac{x \eta^{2}}{2}-\frac{x}{2 v^{2}}
\end{aligned}
$$

and it follows that

$$
\left|P_{u} f_{v}(x)\right|=\left|\int_{-1}^{1} g(\eta) e^{i x \eta^{2} / 2} e^{G(\eta)} d \eta\right| .
$$

We have

$$
|G(\eta)| \leq \frac{x^{2} v^{4}}{4}\left(\frac{2}{v^{2}}\right)^{2}=x^{2} \leq 1
$$

for $|\eta| \leq 1$ and we conclude that

$$
\left|P_{u} f_{v}(x)\right| \geq \int_{-1}^{1} g(\eta) \cos \left(x \eta^{2} / 2\right) e^{G(\eta)} d \eta \geq \int_{-1}^{1} g(\eta) \frac{1}{2} e^{-1} d \eta \geq \frac{1}{2 e} .
$$

Hence $P^{*} f_{v}(x) \geq 1 /(2 e)$ for $0<x<1 / 100$ and $\left\|P^{*} f_{v}\right\|_{2} \geq c>0$ for small $v$. It follows that the estimate $\left\|P^{*} f_{v}\right\|_{2} \leq C\left\|f_{v}\right\|_{H_{s}}$ does not hold for $s<1 / 4$. Hence the statement in Theorem 1 in the case $\gamma=2$ has been proved.

It remains to study the case $\gamma \geq 4$. Take $g$ as above and choose $f$ so that $\widehat{f}(\xi)=g(\xi+N)$ where $N$ denotes a large positive number. Also set $u=u(x)=x /(2 N)$ and assume $2^{-1} N^{1-2 / \gamma} \leq x \leq N^{1-2 / \gamma}$.

Setting $\eta=\xi+N$ we obtain

$$
\begin{aligned}
P_{u} f(x) & =\int e^{i x \xi} e^{i u \xi^{2}} e^{-u^{\gamma} \xi^{2}} g(\xi+N) d \xi \\
& =\int e^{i x(\eta-N)} e^{i u(\eta-N)^{2}} e^{-u^{\gamma}(\eta-N)^{2}} g(\eta) d \eta \\
& =\int g(\eta) e^{i F(\eta)} e^{G(\eta)} d \eta
\end{aligned}
$$

where $F(\eta)=x(\eta-N)+u(\eta-N)^{2}$ and $G(\eta)=-u^{\gamma}(\eta-N)^{2}$. Hence

$$
\begin{aligned}
F(\eta) & =x \eta-x N+u \eta^{2}+u N^{2}-u 2 \eta N \\
& =x \eta-x N+\frac{x}{2 N} \eta^{2}+\frac{x}{2 N} N^{2}-2 \eta \frac{x}{2 N} N \\
& =x \eta-x N+\frac{x}{2 N} \eta^{2}+\frac{x N}{2}-x \eta \\
& =\frac{x}{2 N} \eta^{2}-\frac{x N}{2}
\end{aligned}
$$


and it follows that

$$
\left|P_{u} f(x)\right|=\left|\int_{-1}^{1} g(\eta) e^{i x \eta^{2} /(2 N)} e^{G(\eta)} d \eta\right|
$$

We have

$$
|G(\eta)| \leq u^{\gamma}(2 N)^{2}=\frac{x^{\gamma}}{2^{\gamma} N^{\gamma}} 4 N^{2} \leq N^{(1-2 / \gamma) \gamma} N^{2-\gamma}=1
$$

for $|\eta| \leq 1$ and we conclude that

$$
\left|P_{u} f(x)\right| \geq \int_{-1}^{1} g(\eta) \cos \left(x \eta^{2} /(2 N)\right) e^{G(\eta)} d \eta \geq \int_{-1}^{1} g(\eta) \frac{1}{2} e^{-1} d \eta \geq \frac{1}{2 e} .
$$

Hence $P^{*} f(x) \geq 1 /(2 e)$ for $2^{-1} N^{1-2 / \gamma} \leq x \leq N^{1-2 / \gamma}$ and it follows that

$$
\left\|P^{*} f\right\|_{2} \geq c N^{(1-2 / \gamma) / 2}=c N^{1 / 2-1 / \gamma}
$$

On the other hand it is easy to see that $\|f\|_{H_{s}} \leq C N^{s}$, and if (1) holds, one obtains $N^{1 / 2-1 / \gamma} \leq C N^{s}$. We conclude that $s \geq 1 / 2-1 / \gamma$ and the proof of Theorem 1 is complete.

\section{REFERENCES}

1. Dahlberg, B. E. J., and Kenig, C. E., A note on the almost everywhere behavior of solutions to the Schrödinger equation, pp. 205-209 in: Harmonic Analysis (Minneapolis 1981), Lecture Notes in Math. 908, Springer, Berlin 1982.

2. Sjölin, P., Global maximal estimates for solutions to the Schrödinger equation, Studia Math. 110 (1994), 105-114.

3. Sjölin, P., Maximal estimates for solutions to the nonelliptic Schrödinger equation, Bull. London Math. Soc. 39 (2007), 404-412.

4. Sjölin, P., and Soria, F., Estimates for multiparameter maximal operators of Schrödinger type, Manuscript.

5. Stein, E. M., Harmonic Analysis. Real-variable Methods, Orthogonality, and Oscillatory Integrals, Princeton Mathematical Series 43, Princeton Univ. Press, Princeton, NJ 1993.

DEPARTMENT OF MATHEMATICS

ROYAL INSTITUTE OF TECHNOLOGY

S-100 44 STOCKHOLM

SWEDEN

E-mail: pers@math.kth.se 